\title{
An Approach to the Spacecraft Functional Stability with Changes of Inertia Moments
}

\author{
Nguyen Quang Vinh \\ Department of Radio-Electronic, Institute of Science and Technology, 17 Hoang Sam Road, Cau Giay District, Hanoi, Viet Nam.
}

\begin{abstract}
The paper considers a comprehensive solution providing functional stability of the spacecraft with changes of its moments of inertia. The mathematical description of the spacecraft APSS (angular position stabilization system) in the linear and discrete forms was developed. The process of obtaining spacecraft APSS diagnostic models for the particular kind and class of failure was shown. The possibility to diagnose and restore functionality spacecraft APSS at the systemic level was proved, using the parametric adjustment of regulator coefficients.
\end{abstract}

Key words: Spacecraft, angular position stabilization system, fault tolerance, diagnosis, failure, restore, parametric adjustment.

\section{Introduction}

Analysis of the progress in the space exploration indicates an increase in the requirements for the weight and size characteristics, lifetime and functionality of a small spacecraft. These requirements lead to the complication of the spacecraft APSS. The complexity makes the system more sensitive to the failure. The failure changes the behavior of the control object (spacecraft) so that the system no longer satisfies the goal. Failures in the system can occur due to aging ingredients and depreciation or due to errors in configuring and operating the system. Also, the failure may occur due to changes in environmental conditions. In any case, the failure is a major cause of changes in the system structure or its parameters, which leads to the poor performance or even loss of the system functionality.

Use of the traditional methods associated with the construction of the majority circuit parry of failures results in a significant increase of the power, mass and dimension, the cost of parameters of the spacecraft. Therefore, researches related to the construction of systems with active fault tolerance, were recently developed. They are based on the principles of

Corresponding author: Nguyen Quang Vinh, doctor, main research field: control. E-mail: vinhquang2808@yahoo.com. diagnosis and recovering of the functional system state. Currently, various methods and models of fault tolerance of separate functional blocks of the spacecraft APSS were developed [1, 2]. However, these methods do not consider the APSS fail as a whole, at the systemic level. Therefore, the actual problem is a systematic approach to the development of an APSS active fault tolerant.

In this paper, one considers a systematic approach to a place of failure associated with the change in the spacecraft moment of inertia. One cause of this failure is an incomplete disclosure of spacecraft solar panels. An incomplete disclosure, as presented in the various reports, may be due to some structural defect in the mechanism of disclosure, strain solar surface, mounting damaging one of the solar panels on startup, etc. [3]

\section{Mathematical Description of the Spacecraft APSS}

Let one consider equations of motion of the spacecraft related to the center of mass. One of the most characteristic features of the spacecraft as the control object is the impact on its angular position internal moment's movements resulting from the relative motions of the device parts. There are two possible following cases: 
Internal moments are absent or compared with the external moments. They are so small that their effect on the angular motion can be ignored;

Internal moments are commensurate with the external or they are the main cause of the angular motion of the vehicle, so that they can not be ignored.

The second case is often used to control the angular position of the spacecraft.

The principle of using the reaction wheel as the device for creating control moments based on the law of angular momentum conservation. If the flywheel is rotated by the motor in one direction, the spacecraft will rotate in the opposite direction.

Next, consider the spacecraft with control executive bodies in form of contactless torque DC motors. The rotor with permanent magnets is located at the maximum diameter and performs the function of centrifugal mass (reaction wheel).

One will take the circuit shown in Fig. 1 as the installation diagrams of four RW (reaction wheels).

In addition, one assumes that the angular speed of rotation is much greater than the angular velocity of angular motion. RW's rotation does not change the position of the spacecraft center of mass and the total moment of inertia.

Dynamics equations for this object are obtained from the basis Lagrange equations of the second kind [4].

Let one consider linearized equation for the closed APSS of the spacecraft.

The functional diagram of the system is shown in Fig. 2.

Linearized equations for the APSS can be written as Eq. (1):

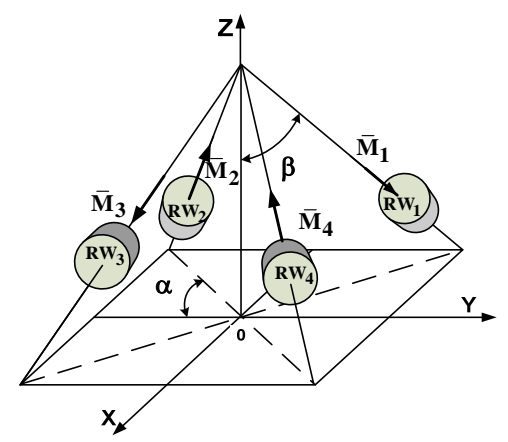

Fig. 1 Installation diagram of RW on the spacecraft.

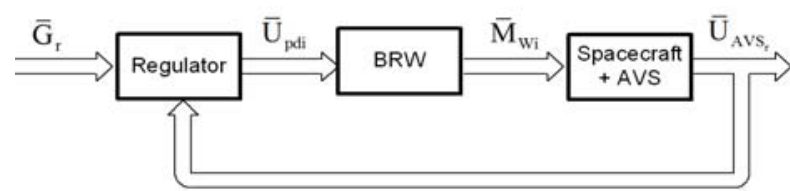

Fig. 2 Functional diagram of the closed-loop system.

$$
\begin{aligned}
& \dot{\omega}_{x}(t) \cdot I_{x x}+4 \dot{\omega}_{x}(t) I_{M}+I_{z z} \omega_{y}(t) \omega_{z . r}-I_{y y} \omega_{y}(t) \omega_{z . r}-I_{y y} \omega_{y . r} \omega_{z}(t)+I_{z z} \omega_{y . r} \omega_{z}(t)+I_{M} \omega_{y}(t) \cdot a_{33} \cdot k_{2 x}+ \\
& +I_{M} \omega_{z}(t) \cdot a_{32} \cdot k_{3 x}+I_{M} k_{4 x} \cdot\left(\omega_{R W 1}(t)-\omega_{R W 2}(t)++\omega_{R W 3}(t)-\omega_{R W 4}(t)\right)=M_{x} \text {; } \\
& \dot{\omega}_{y}(t) \cdot I_{y y}+4 \dot{\omega}_{y}(t) I_{M}+I_{M} a_{32}\left(\dot{\omega}_{R W 1}(t)-\dot{\omega}_{R W 2}(t)+\dot{\omega}_{R W 3}(t)-\dot{\omega}_{R W 4}(t)\right)+I_{x x} \omega_{x}(t) \omega_{z . r}-I_{z z} \omega_{x}(t) \omega_{z . r}+ \\
& +I_{z z} \omega_{x . r} \omega_{z}(t)+I_{x x} \omega_{x . r} \omega_{z}(t)+\omega_{x}(t) \cdot k_{1 y}+\mathrm{I}_{\mathrm{M}} k_{4 y} \cdot\left(-\omega_{R W 1}(t)+\omega_{R W 2}(t)-\omega_{R W 3}(t)+\omega_{R W 4}(t)\right)=M_{y} \text {; } \\
& \dot{\omega}_{z}(t) \cdot I_{z z}+4 \dot{\omega}_{z}(t) I_{M}+I_{M} a_{33}\left(\dot{\omega}_{R W 1}(t)-\dot{\omega}_{R W 2}(t)+\dot{\omega}_{R W 3}(t)-\dot{\omega}_{R W 4}(t)\right)+I_{y y} \omega_{x}(t) \omega_{y . r}-I_{x x} \omega_{x}(t) \omega_{y . r}+ \\
& +I_{x x} \omega_{x . r} \omega_{y}(t)+I_{y y} \omega_{x . r} \omega_{y}(t)+\omega_{x}(t) \cdot k_{1 z}+\mathrm{I}_{\mathrm{M}} k_{4 y} \cdot\left(-\omega_{R W 1}(t)+\omega_{R W 2}(t)-\omega_{R W 3}(t)+\omega_{R W 4}(t)\right)=M_{z} \text {; } \\
& I_{M}\left(a_{32} \dot{\omega}_{y}(t)+a_{32} \dot{\omega}_{z}(t)+\dot{\omega}_{R W 1}(t)+a_{13} k_{15} \omega_{x}(t)-a_{23} k_{25} \omega_{y}(t)+a_{33} k_{35} \omega_{z}(t)-\right. \\
& \left.-\mathrm{k}_{45} \omega_{R W 1}(t)-a_{13} k_{55} G_{x}(t)+a_{23} k_{65} G_{y}(t)-a_{23} k_{95} \int \omega_{y}(t) d t+a_{33} k_{105} \int \omega_{z}(t) d t\right)=0 \text {; } \\
& I_{M}\left(-a_{32} \dot{\omega}_{y}(t)-a_{32} a_{33} \dot{\omega}_{z}(t)+\dot{\omega}_{R W 2}(t)+a_{13} k_{55} G_{x}(t)+a_{23} k_{65} G_{y}(t)-a_{33} k_{75} G_{z}(t)-\right. \\
& \left.-\mathrm{a}_{13} k_{85} \int \omega_{x}(t) d t-a_{23} k_{95} \int \omega_{y}(t) d t\right)=0 \text {; } \\
& I_{M}\left(a_{32} \dot{\omega}_{y}(t)+a_{32} a_{33} \dot{\omega}_{z}(t)+\dot{\omega}_{R W 3}(t)-a_{13} k_{15} \omega_{x}(t)+a_{23} k_{25} \omega_{y}(t)+a_{33} k_{35} \omega_{z}(t)-\mathrm{k}_{45} \omega_{R W 3}(t)+\right. \\
& \left.+a_{13} k_{55} G_{x}(t)-a_{23} k_{65} G_{y}(t)-a_{33} k_{75} G_{z}(t)-\mathrm{a}_{13} k_{85} \int \omega_{x}(t) d t+a_{23} k_{95} \int \omega_{y}(t) d t\right)=0 ; \\
& I_{M}\left(-a_{32} \dot{\omega}_{y}(t)-a_{32} a_{33} \dot{\omega}_{z}(t)+\dot{\omega}_{R W 4}(t)+a_{13} k_{15} \omega_{x}(t)+a_{23} k_{25} \omega_{y}(t)+a_{33} k_{35} \omega_{z}(t)-\right. \\
& \left.-\mathrm{k}_{45} \omega_{R W 4}(t)-a_{13} k_{55} G_{x}(t)-a_{23} k_{65} G_{y}(t)-a_{23} k_{95} \int \omega_{y}(t) d t+a_{33} k_{105} \int \omega_{z}(t) d t\right)=0 \text {, }
\end{aligned}
$$


where,

$$
\begin{aligned}
& \bar{I}=\left[\begin{array}{ccc}
I_{x x} & 0 & 0 \\
0 & I_{y y} & 0 \\
0 & 0 & I_{z z}
\end{array}\right] \text {-spacecraft tensor of inertia; } \\
& \bar{I}_{M i}=\left[\begin{array}{ccc}
I_{M} & 0 & 0 \\
0 & I_{M} & 0 \\
0 & 0 & I_{M}
\end{array}\right], \mathrm{i}=1 \ldots 4-\mathrm{RW} \text { tensor of }
\end{aligned}
$$

inertia;

$$
M_{i}=\left[\begin{array}{ccc}
\cos \alpha & \cos \beta & \sin \alpha \sin \beta \\
-\sin \alpha & \cos \alpha \cos \beta & \cos \alpha \sin \beta \\
0 & -\sin \beta & \cos \beta
\end{array}\right]
$$

direction cosine matrix characterizing the position of the $\mathrm{i}$-th RW in the coupled system of coordinates; $\omega_{\mathrm{x}}$, $\omega_{\mathrm{y}}, \quad \omega_{\mathrm{z}}$-spacecraft angular speed relatively corresponding axes; $\omega_{\mathrm{RWi}}, \mathrm{I}=1.4-\mathrm{I}$-th RW angular speed; $\mathrm{k}_{\mathrm{j} 5}, \mathrm{j}=1 \ldots 10$ - coefficients that are functions of $\mathrm{K}_{\mathrm{p}}, \mathrm{K}_{\mathrm{d}}$-regulator coefficients, $\mathrm{K}_{\text {dus }}$-AVS coefficient and $\mathrm{K}_{\mathrm{um}}, \mathrm{T}_{\mathrm{M}}$ - motor coefficients; $\mathrm{G}_{\mathrm{x}}, \mathrm{G}_{\mathrm{y}}, \mathrm{G}_{\mathrm{z}}$ - control voltages on the input of system. One defines the state variables, control vectors and output signals for further transition from the equations describing the motion of the functional elements to the system of equations related to the spacecraft input and output signals:

$$
\begin{aligned}
u^{T}(t)= & {\left[\begin{array}{lll}
G_{x}(t) & G_{y}(t) & G_{z}(t)
\end{array}\right] } \\
x^{T}(t)= & {\left[\begin{array}{ll}
\int \omega_{x}(t) d t ; \int \omega_{y}(t) d t ; \int \omega_{z}(t) d t ; \\
\omega_{x}(t) ; \omega_{y}(t) ; \omega_{z}(t) ; \omega_{R \mathrm{~W} 1}(t) ; \omega_{R \mathrm{~W} 2}(t) ; \\
\omega_{R \mathrm{~W} 3}(t) ; \omega_{R \mathrm{~W} 4}(t)
\end{array}\right] }
\end{aligned}
$$

Measurement and production control actions are performed with a time interval $\mathrm{T}_{0}$ because the system contains the analog-to-digital converter and the digital-to-analog converter and all procedures of processing, transformation and analysis are carried out in a microcontroller. One uses the representation of derivatives in the form of finite differences in describing the behavior of the continuous part of the automatic control object at time $\mathrm{kT}_{0}, \mathrm{k}=0,1 \ldots$ Then one can obtain the system of equations describing the behavior of the nominal APSS in the discrete form.

\section{Procedure for Selecting the Best Model}

One will make parameterization of the kinds of faults for the control object spacecraft. Under the kind of functional element with fault, one means any event associated with the deviation of its static and dynamic characteristics from its nominal value.

Spacecraft set of kinds of faults includes the following elements: $d_{\mathrm{sp} 1}, \mathrm{~d}_{\mathrm{sp} 2}$-respectively, the kinds of faults, characterized by a decrease and increase of the moment of inertia $\mathrm{I}_{\mathrm{xx}} ; \mathrm{d}_{\mathrm{sp} 3}, \mathrm{~d}_{\mathrm{sp} 4}$-respectively, the kinds of faults, characterized by a decrease and increase of the moment of inertia $I_{y y} ; d_{s p 5}$, $\mathrm{d}_{\mathrm{sp} 6}$ - respectively, the kinds of faults, characterized by a decrease and increase of the moment of inertiaI $I_{z z}$.

Many types of spacecraft faults can be represented by three classes: $\alpha_{\mathrm{sp} 1}$ - class of spacecraft faults, including the kinds of faults $\mathrm{d}_{\mathrm{sp} 1}, \mathrm{~d}_{\mathrm{sp} 2}$ and characterized by the parameter $\widetilde{I}_{x x} ; \alpha_{\text {sp } 1}$-class of spacecraft faults, including the kinds of faults $\mathrm{d}_{\mathrm{sp} 3}, \mathrm{~d}_{\mathrm{sp} 4}$ and characterized by the parameter $\widetilde{I}_{y y} ; \alpha_{\mathrm{sp} 3}$-class of spacecraft faults, including the kinds of faults $d_{\mathrm{sp} 5}, \mathrm{~d}_{\mathrm{sp} 6}$ and characterized by the parameter $\widetilde{I}_{z z}$.

One associates spacecraft direct diagnostic features with deviations of ARS output signals in compiling DFM (diagnostic functional models) for the classes of spacecraft faults. DFM links of direct diagnostic features for classes of spacecraft faults $\alpha_{\mathrm{sp} 1}$ and $\alpha_{\mathrm{sp} 2}$ with their deviations from their estimations of main central reservation system output signals established along axes of the spacecraft associated coordinate system, have the form:

$$
\begin{aligned}
& \Delta x_{4}[k+1]=\Delta x_{4}[k]+T_{0}\left(\sum_{j=1}^{10} \Delta I_{x x} k_{j}\left(I_{x x}, I_{y y}, I_{z z}, K_{d}, K_{p}\right) \tilde{x}_{j}+\sum_{r=1}^{3} \Delta I_{x x} k_{r}\left(I_{x x}, I_{y y}, I_{z z}, K_{d}, K_{p}\right) G_{r}\right) \\
& \Delta x_{5}[k+1]=\Delta x_{5}[k]+T_{0}\left(\sum_{j=1}^{10} \Delta I_{x x} k_{j}\left(I_{x x}, I_{y y}, I_{z z}, K_{d}, K_{p}\right) \tilde{x}_{j}+\sum_{r=1}^{3} \Delta I_{x x} k_{r}\left(I_{x x}, I_{y y}, I_{z z}, K_{d}, K_{p}\right) G_{r}\right) \\
& \Delta x_{6}[k+1]=\Delta x_{6}[k]+T_{0}\left(\sum_{j=1}^{10} \Delta I_{x x} k_{j}\left(I_{x x}, I_{y y}, I_{z z}, K_{d}, K_{p}\right) \tilde{x}_{j}+\sum_{r=1}^{3} \Delta I_{x x} k_{r}\left(I_{x x}, I_{y y}, I_{z z}, K_{d}, K_{p}\right) G_{r}\right)
\end{aligned}
$$


Let one analyze these DFM. The evidence is reviewed by using the criteria of the DFM structure and signal diagnosability. It is not difficult to show that is provided the condition of structural diagnosability, and the unique determination of direct diagnostic characteristics of failures classes is provided with non-zero control and angular momentum generated by $\mathrm{RW}$, as well as with non-zero spacecraft speeds. Consequently, these models are possible to use for the purpose of deep system diagnosing, the result of which is to determine the failures characteristics. Failures characteristics, such as the detection time, place, class and kind, obtained as a result APSS diagnosis. They are the foundation for the next phase for active fault tolerance - parrying failures by using the available in the object structural, functional and information redundancy [5].

\section{Development of Procedures for the Restoring Functionality of the Spacecraft APSS}

According to the systems approach to the active fault tolerance, the development of parrying failures procedures is carried out by the APSS research, which includes the following steps: identification for each failure mode every possible variants of its parrying through available excess resources; the allocation from the whole set of options the parrying subsets that characterize element and system level fault tolerance; establishing for each kind of failure the relative priority, necessary for the formation procedures for the effective use of appropriate redundancies.

According to Eq. (2) the DFM for this kind of failure include transfer coefficients PD controller. If one allows these coefficients change according to the change of parameters $\tilde{\mathrm{I}}_{\mathrm{xx}}, \tilde{\mathrm{I}}_{\mathrm{yy}}, \tilde{\mathrm{I}}_{\mathrm{zz}}$, it is possible to parry these kinds of failures at the system level by adjusting the regulator coefficients. Thus, there is a possibility of parametric adjustment for changing the parameters of system components (transmission coefficients, voltages, etc.) in order to parry kinds of failure detected in the object.

Fig. 3 shows the connection diagram of the parametric adjustment block.

For example, a parameter adjustment of the regulator matrix of transfer coefficients $\delta \mathrm{kp}$ (k) guarantees asymptotic stability of the spacecraft motion; it is based on the following expressions:

$$
\begin{gathered}
\mathrm{Kp}(\mathrm{k})=\mathrm{Kp}+\delta \mathrm{kp}(\mathrm{k}) ; \delta \mathrm{kp}(\mathrm{k})= \\
\delta \mathrm{k}^{*} \mathrm{p}(\mathrm{k})+\delta \mathrm{kp} . \mathrm{st}
\end{gathered}
$$

where $\delta \mathrm{k}^{*} \mathrm{p}(\mathrm{k})$ is the component to compensate the influence of parametric perturbations; $\delta \mathrm{kp} . \mathrm{st}$ is the component that provides the asymptotic stability of the object perturbed motion.

The expressions for calculating $\delta \mathrm{k}^{*} \mathrm{p}(\mathrm{k})[6]$ are as follows:

$$
\begin{aligned}
& \Delta K_{p}^{*}(k)=\left(B+\Delta \hat{B}\left(\Delta \lambda_{1}\right)\right)^{-1} n(k)(C * \widetilde{x}(k)-k(k))^{-1} ; \\
& n(k)=\Delta \hat{F}\left(\Delta \lambda_{i}\right) f(k)-\Delta \hat{B}\left(\Delta \lambda_{i}\right) K_{p}(k) C^{*} \widetilde{x}(k)+ \\
& +\Delta \hat{B}\left(\Delta \lambda_{i}\right) K_{p} g(k)+\hat{b}_{0}\left(\Delta \lambda_{i}\right),
\end{aligned}
$$

where $\mathrm{B}$ is the control matrix of spacecraft APSS, $\Delta \hat{B}\left(\Delta \lambda_{i}\right), \Delta \hat{F}\left(\Delta \lambda_{i}\right), \hat{b}_{0}\left(\Delta \lambda_{i}\right)$, - matrices and vectors describing spacecraft dynamics of change in the deviation relative to the nominal; $C^{*}$ - the matrix consisting of the transmission coefficients of sensors.

Eq. (4) for calculating values $\delta \mathrm{kp}$.st (k) is determined based on the discrete analog of the Lyapunov second method:

$$
\delta \mathrm{kp} . \mathrm{st}=0
$$

$$
\Delta K_{p . s t}(k)=-2\left(B+\Delta \hat{B}\left(\Delta \lambda_{i}\right)\right)^{-1} G \Delta x(k) \varepsilon^{-1}(k)
$$

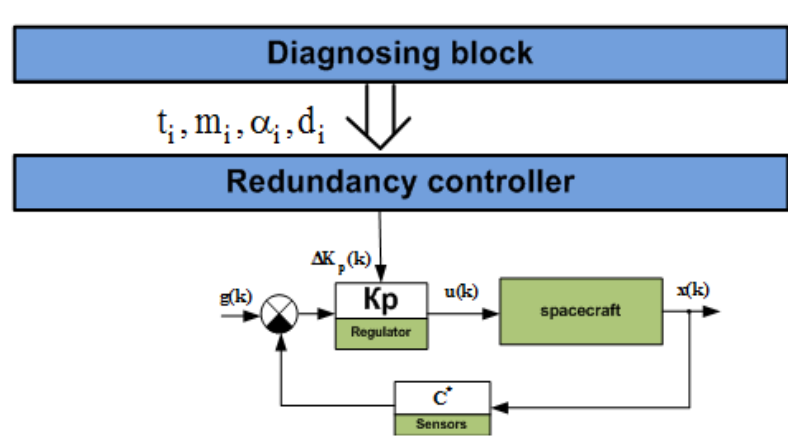

Fig. 3 Scheme of parametric adjustment realization. 


\section{Conclusions}

The research resulted in received mathematical models of spacecraft APSS. They are used as a basis for the formation of diagnostic functional models for the failure place related with the change of spacecraft moments of inertia. The impact of the controller parameters on the spacecraft APSS parameters was shown. Possible recovery procedures of the APSS functional state are developed on the basis of the system (2) at the system level. It is shown that by using Eqs. $(3,4)$ it is possible to carry out a parametric adjustment of regulator coefficients that provides the asymptotic stability of the spacecraft angular motion.

\section{References}

[1] Gavrilenko, O. I., Luchenko, O. A., and Resnikova, O. V. 2007. "A Research on the Diagnostic Guaranty for the
Stability System of Spacecrafts.” Radio-Electronical and Computer Systems 7 (26): 134-9.

[2] Kulik, A. C., Firsov, C. N., and Taran, A. N. 2010. "Diagnosis of the Functional State of the Orientation Electric Flywheel System of the Angular Position of a Microsatellite." Radio-Electronic and Computer Systems 4 (45): 82-90.

[3] Medvedchikov, D. A. 2006. "The International Market of Space Insurance: History, Dynamics, Trends." Insurance Business Number 1: 41-52.

[4] Nguyen, Q. V. 2006. "Influences of Nonlinearities in the Control Circle of the Self Guidance on a Maneuvering Target." Actual Problems of Russian Cosmonautics. Moscow: Materials of 30th Academic Readings on Cosmonautics.

[5] Pupkov, K. A., and Vinh, N. Q. 2006. Analysis of the Self Guidance Precision on a Maneuvering Target with Nonlinearities in the Control Circles. Moscow: Science Intensive Technology.

[6] Firsov, C. N. 2005. "The Guaranty of Active Fault Tolerance of Pneumatic Servomotor UAVs." Ph.D. thesis, National Aerospace University. 